\title{
MRT verbessert nicht die Demenzvorhersage
}

Fragestellung: Kann der Befund aus der Magnetresonanztomografie (MRT) die Vorhersage einer Alzheimer-Demenz im Vergleich zu den konventionellen Risikofaktoren verbessern?

Hintergrund: Die Prävalenz der Alzheimer-Demenz wird sich alle 20 Jahre verdoppeln. Da die Krankheit meist im höheren Lebensalter auftritt und fünf bis sieben Jahre bis zum Tod dauert, würde eine Verzögerung der Inzidenz um zwei Jahre die Prävalenz deutlich reduzieren. Es ist daher wichtig, das Risiko einer künftigen Demenz zu beurteilen, um die Interventionen für Hochrisikopopulationen zielgerichteter planen zu können und die Kosten für unnötige diagnostische Tests zu reduzieren.

Zur Vorhersage einer Demenz bieten sich vor allem populationsbasierte Studien an. Während Alter der universelle Risikofaktor für die Demenz ist, haben konventionelle soziodemografische Modelle Faktoren wie die Ergebnisse neuropsychologischer Tests, subjektive Gedächtnisprobleme, Schulbildung,

Geschlecht, Depression, zerebro- und kardiovaskuläre

Stephan B, Tzourio C, Auriacombe $S$ et al. Usefulness of data from magnetic resonance imaging to improve prediction of dementia: population based cohort study. BMJ 2015; 350: h2863. doi: 10.1136/bmj.h2863 Erkrankungen und Risikofaktoren, Alltagskompetenz und genetische Faktoren wie APO E aufgenommen. Die Vorhersagekraft dieser Modelle könnte durch bildgebende Methoden erhöht wer- den, da es bei der Demenz zu charakteristischen strukturellen Veränderungen wie Hippocampusatrophie, mediotemporale Atrophie und Marklagerläsionen kommt.

Patienten und Methodik: In der populationsbasierten Kohortenstudie wurde in Dijon Freiwillige ohne Demenz im Alter von 65 bis 80 Jahren untersucht. Es wurden 1.721 MRT-Aufnahmen gemacht und die Entwicklung der Studienteilnehmer über 0,6 bis 10,6 Jahre verfolgt. Endpunkt war die Entwicklung einer Demenz (jeder Ätiologie und Alzheimer-Demenz).

Ergebnisse: Im Studienverlauf entwickelten 119 Teilnehmer eine Demenz, 84 davon eine Alzheimer-Demenz. Das konventionelle Risikomodell umfasste Alter, Geschlecht, Schulbildung, Kognition, körperliche Fitness, Nikotin, Alkohol, Diabetes, Blutdruck sowie den APO-E-Geotyp und konnte $77 \%$ der Fälle korrekt erfassen. Die Hinzunahme einzelner oder kombinierter MRT-Variablen erhöhte die Erkennungsrate für alle Demenzen oder für die Alzheimer-Demenz nicht. Das MRT, insbesondere das Hippocampusvolumen, war jedoch klinisch nützlich bei der Reklassifikation verschiedener Demenzen, bei der Risikoklassifikation oder bei der Prognoseneinschätzung.

Schlussfolgerungen: Das Ergebnis des MRT-Befundes verbessert die mögliche Früherkennung einer Demenz im Vergleich zu klinischen Risikofaktoren nicht.

\section{- Kommentar von Markus Weih, Nürnberg}

\section{MRT kann bei bereits bestehenden Gedächtnisproblemen hilfreich sein}

Die französischen Epidemiologen haben wie in der Vergangenheit wieder einmal ganze Arbeit geleistet. Das gute Ergebnis für die Praxis: Das Erfassen klassischer kardiovaskulärer Risikofaktoren sowie einiger weiterer, leicht erfassbarer Informationen wie Schulbildung und einfache neuropsychologische Tests sind fast genauso gut wie ein MRT.

Die Studie hat jedoch auch ihre Schwächen: Lebensstil, Ernährung und die Lebenserwartung sind in Frankreich anders und nicht einfach auf andere Länder übertragbar. Generell gilt, dass die meist gesunden und gesund lebenden Freiwilligen in solchen Kohortenstudien nicht einfach mit Patienten - selbst wenn nur eine leichte kognitive Störung vorliegt - zu vergleichen sind.

Dennoch: Das Ergebnis der Studie ist gut, für den niedergelassenen Nervenarzt und sogar für den Hausarzt. Es gibt wenige Krankheiten, vor denen Menschen soviel Angst haben wie vor dem geistigen Verfall bei der Demenz. Jeder kennt die Sorgen von Patienten, die Angehörige haben oder gepflegt haben und besorgt in die Sprechstunde kommen, um "sich testen" oder beraten zu lassen. Die gängige Praxis der allgemeinen Gesundheitsanamnese, dann der Durchführung neuropsychologischer Tests, der Rat zu Kontrolle und Modifikation allgemeiner Herzkreislaufrisiken sowie der Rat zu kognitivem Training ist also wahrscheinlich gar nicht so schlecht.

Welche Indikation bleibt dann für das MRT? Wie in den S3Leitlinien natürlich für Patienten, die schon Gedächtnisprobleme haben, zur Einschätzung des Ausmaßes der Mikroangiopathie beziehungsweise der vaskulären Läsionen, zur Beurteilung der globalen und Hippocampusatrophie und wenn etwas nicht ins Muster der häufigsten Demenz, also der Alzheimer-Demenz passt. Hier wäre es hilfreich, wenn es noch mehr automatische Klassifikationen gäbe und die MRT-Befunde standardisierter wären, als es oft noch die Praxis ist.

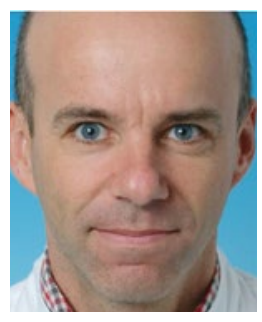

Prof. Dr. med. Markus Weih, Nürnberg

Facharzt für Neurologie, Psychiatrie und Psychotherapie, Nervenärztliche Gemeinschaftspraxis, Nürnberg E-Mail:weih@nervenaerzteallersbergerstrasse.de 